8

16 Key Words: behavioral fault modeling, macro modeling, analog fault simulation, VHDL-AMS

\title{
Behavioral Fault Modeling and Simulation Using VHDL-AMS to Speed-Up Analog Fault Simulation
}

\author{
Y. KILIÇ ${ }^{1}$ AND M. ZWOLIŃSKI ${ }^{2}$ \\ ${ }^{1}$ Allegro MicroSystems Europe Ltd., Stuart House, Station Road, Musselburgh, EH21 7PB, UK \\ ${ }^{2}$ Department of Electronic and Computer Science, University of Southampton, Highfield, Southampton, SO17 1BJ, UK \\ E-mail: yavuz.kilic@ieee.org; mz@ecs.soton.ac.uk
}

Received ; Revised November 2, 2002; Accepted June 11, 2003

Au: Pls. provide received date.

\begin{abstract}
One of the main requirements for generating test patterns for analog and mixed-signal circuits is fast fault simulation. Analog fault simulation is much slower than the digital equivalent. This is due to the fact that digital circuit simulators use less complex algorithms compared with transistor-level simulators. Two of the techniques to speed up analog fault simulation are: fault dropping/collapsing, in which faults that have similar circuit responses compared with the fault-free circuit response and/or with another faulty circuit response are considered equivalent; and behavioral/macro modeling, whereby parts of the circuit are modeled at a more abstract level, therefore reducing the complexity and the simulation time. This paper discusses behavioral fault modeling to speed-up fault simulation for analog circuits.
\end{abstract}

\section{Introduction}

As transistor sizes shrink, integrated circuits (ICs) have been growing in size and functionality. This growth in IC complexity causes testing to become much more difficult. For digital circuits the problem of testing can be simplified by using standard fault models and fast fault simulation. Faults in digital circuits can be modeled as stuck-at, bridging, delay and open faults. These structural faults can then be used to generate functional test patterns. The objective of a test program for digital circuits translates into determining whether or not a fault exists using the smallest possible number of test patterns [1].

A test pattern is evaluated by looking at its fault coverage. All faults detected with a pattern can be dropped from further consideration. Fault simulation is done to assess the fault coverage. There are a number of fault simulation techniques for digital circuits. Serial fault simulation is perhaps the simplest method. For each fault, a "faulty" copy of the circuit with that fault inserted is created. Then, all the faulty copies of the circuits along with the fault-free one are simulated with the test pattern. If the output of a faulty circuit differs from the fault-free output, that fault is considered to be detectable.

Another fault simulation technique for digital circuits is concurrent fault simulation [2]. The differences between the faulty and fault-free circuit behaviors might be relatively small. Therefore, in concurrent fault simulation the aim is to avoid redundant element evaluation when the faulty and fault-free behaviors are the same hence reducing the computational effort.

Analog and mixed-signal fault simulation has been limited to the serial technique. Faster digital fault simulation methods are not easily applied to analog circuits and/or mixed-signal circuits, because faults do not affect the behavior of circuit nodes in a binary manner.

One way to speed-up fault simulation for analog and mixed-signal circuits is to use behavioral/macro models, where parts of the circuit are modeled at a more abstract level, reducing the complexity and hence the simulation time. Characterizing behavioral fault models requires low-level simulations and is therefore not applicable in every case. There are three situations in which behavioral fault modeling might be of benefit, however. First, if circuit blocks were reused, lowlevel fault simulations would not have to be repeated. 
Second, at the system design stage, information about possible faults and how their effects might be propagated can be used to insert optimal test structures. Third, actual faults can be modeled at a low level (analog or digital), while neighboring circuit blocks can be modeled behaviorally, again reducing the overall simulation time.

In this paper behavioral fault simulation for analog CMOS circuits is investigated. The structure of the rest of the paper is as follows. First, macro modeling for analogue circuits is presented. Then behavioral modeling is discussed with a case study. In Section 4, behavioral modeling using Hardware Description Languages (HDLs) is summarized. In Section 5, a behavioral fault model is developed in VHDL-AMS [3] for an opamp circuit operating in inverting amplifier configuration and the model is simulated using the hAMSter VHDLAMS simulator [4]. Simulation results are given in Section 6. Finally, in Section 7 some conclusions are drawn.

\section{Macromodels for Analogue Circuits}

Simulation at the transistor level for analog circuits is computationally very expensive. One way to reduce this high simulation cost is to partition a large analog circuit into smaller functional blocks such as opamps (operational amplifiers) and to replace each functional block with its macromodel or to describe each block using mathematical equations (a behavioral model). This solution is sometimes called hierarchical fault simulation [5].

The word macromodel usually refers to a compact representation of a circuit that captures those features that are useful for a particular purpose while discarding redundant information [6]. Macromodels developed for SPICE-like simulators are basically electrical networks containing devices such as voltage-controlled voltage sources, instead of the full transistor network, and with fewer nodes than the original circuit.

Many circuits are designed in a modular style, in which functional units are connected to achieve design specifications. The behavior of the whole circuit is determined by how the individual units interact with each other, while what happens inside each is unimportant in terms of capturing the input-output relationship for the entire circuit. The accuracy of a macromodel must, therefore, be defined in terms of how closely its inputoutput behavior matches that of the original unit [6].
Since the early 1970s, a number of macromodels 111 have been developed mainly for integrated operational 112 amplifier circuits (opamps) [5,7]. Boyle et al. presented 113 a macromodel for integrated bipolar opamp circuits [8]. 114 This macromodel was six times less complex (in terms $\mathbf{1 1 5}$ of the node count) than the original opamp circuit, and $\mathbf{1 1 6}$ the simulation time was an order of magnitude faster $\mathbf{1 1 7}$ than the device-level model.

118

The derivation of component values for the Boyle 119 macromodel is not, however, straightforward. Some pa- $\mathbf{1 2 0}$ rameters are modeled using unbalanced input devices $\mathbf{1 2 1}$ and other parameters interact. Therefore, a modular ap- 122 proach was suggested [9], in which a macromodel was $\mathbf{1 2 3}$ derived simply from the published data sheets. Individ- $\mathbf{1 2 4}$ ual parameters were modeled separately and the results $\mathbf{1 2 5}$ combined to provide the output response. Since the pa- $\mathbf{1 2 6}$ rameters were separated they did not interact and only $\mathbf{1 2 7}$ those required were included.

128

Recent research has focused on how to capture the $\mathbf{1 2 9}$ effect of a fault in an analogue circuit within its macro- $\mathbf{1 3 0}$ model [1, 3, 10]. The fault macromodeling problem 131 was formulated in terms of deriving the macro param- 132 eter set, $B$, based on the performance parameter set, 133 $P$ (gain, the bandwidth, samples on the frequency or $\mathbf{1 3 4}$ time response curves, etc.) of the transistor-level faulty $\mathbf{1 3 5}$ circuit [5]. The accuracy of the macromodel was eval- $\mathbf{1 3 6}$ uated by checking the consistency of the performance 137 parameter set, $P$, between the transistor-level circuit $\mathbf{1 3 8}$ and the macromodel.

Two steps are needed to obtain the macromodel for $\mathbf{1 4 0}$ a functional block within an analog circuit [5]:

1. Perform transistor level fault simulation for each $\mathbf{1 4 2}$ faulty circuit to obtain the value of the performance 143 parameter set $P$.

144

2. Map each performance parameter set $P$ to the cor- $\mathbf{1 4 5}$ responding macro parameter set, $B$. This is referred 146 to as parameter mapping.

147

It was assumed that the transistor-level fault list is given $\mathbf{1 4 8}$ and the macromodel structure and the performance pa- 149 rameter set, $P$, to be matched are predetermined by the $\mathbf{1 5 0}$ circuit designer.

151

There are several ways to do parameter mapping. 152 One simple approach is based on analytical design 153 equations that express the macro parameter set, $B$, as $\mathbf{1 5 4}$ analytical functions of the performance parameter set, $\mathbf{1 5 5}$ $P$, and the value of $B$ is derived by function evalua- $\mathbf{1 5 6}$ tion. As analog ICs get more complex, this approach 157 is becoming more difficult. Another simple approach $\mathbf{1 5 8}$ 
159 is to build an empirical mapping function, $B=F(P)$,

160 based on a large number of data pairs $(P, B)$, referred

161 to as the training set [5]. Usually the training set is 162 generated by randomly selecting $M$ out of the $N$ per163 formance parameter sets for the faulty circuits obtained 164 by transistor-level simulation and then the value of the 165 macro parameter set $B$ for each selected $P$ is derived. 166 The derivation of each data pair usually requires mul167 tiple runs of the macromodel-level simulation [3].

168 Macromodeling in general and fault macromodeling 169 using SPICE-like languages in particular have, nev170 ertheless, been shown to be very difficult [1, 5-20]. 171 Therefore, another easier and perhaps more efficient 172 way of modeling analog circuits at a higher level is 173 necessary.

\section{3. Behavioral Modeling}

175 A behavioral model describes a circuit block in terms 176 of mathematical equations modeling the functionality 177 of the block, for example, in terms of the input-output 178 relationship. Behavioral modeling has been used for 179 speeding up analog simulation in general [21] and ana$180 \log$ fault simulation in particular $[1,10,20,22]$. In one 181 approach, analog circuits were modeled behaviorally in 182 the C programming language [21]. Broyden's method 183 [23] was used to formulate and solve the model equa184 tions in a custom simulator. The main drawback of this 185 work is that since the technique does not require deriva186 tives it cannot be used for small-signal analysis.

Chang et al. [10] presented a behavioral fault model derived from a macromodel of a CMOS operational amplifier from the IEEE Mixed-Signal Benchmark Suite [24] (Fig. 1). The "faulty" macromodel was de-

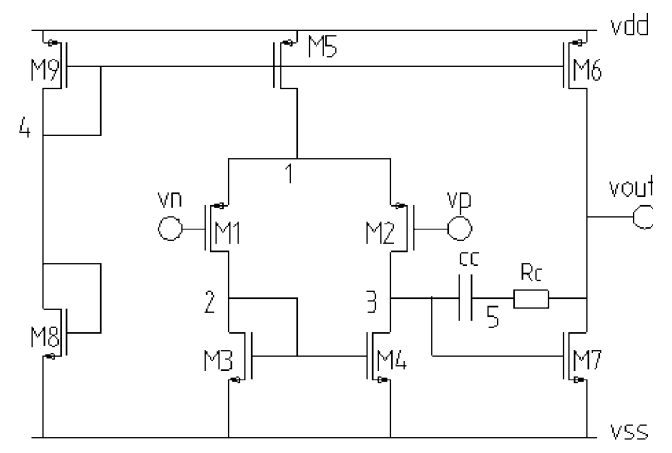

Fig. 1. The 2-stage CMOS Miller opamp used in [10] for behavioral fault modeling. (a)



(b)

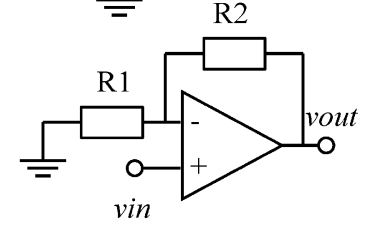

(c)

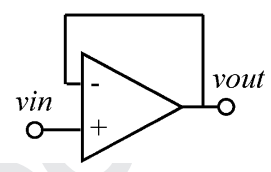

Fig. 2. Three different configurations used in [10] for the benchmark circuit given in [24]: (a) Inverting amplifier, (b) non-inverting amplifier, and (c) unity gain buffer.

veloped using DC-sweep analysis. The DC behavior 191 of the benchmark opamp operating in inverting, non- 192 inverting and unity gain amplifier configurations was 193 first investigated under different fault conditions, as $\mathbf{1 9 4}$ shown in Fig. 2. Single transistor catastrophic faults, 195 bridging/short and nearly open faults, and paramet- 196 ric faults with $W$ (channel width), $L$ (channel length) 197 and $V_{\mathrm{TH}}$ (threshold voltage) varied by $\pm 10 \%$ were 198 used for each transistor. Then an attempt was made 199 to group the different faulty behaviors. By comparing $\mathbf{2 0 0}$ the fault-free offset voltage measured at the inputs of 201 the opamp operating in one of the three configurations $\mathbf{2 0 2}$ with the equivalent faulty circuits, four different equiv- 203 alent fault types were derived [10]: M4 drain-to-gate 204 short (Type I), M5 drain-to-source short (Type II), M7 205 drain open (Type III), and M5 drain-to-source short 206 (Type IV). The first three fault types were found for the $\mathbf{2 0 7}$ opamp operating in the inverting configuration, where 208 the Type IV fault group was found for the non-inverting $\mathbf{2 0 9}$ configuration.

210

The input offset voltage (measured between the pos- 211 itive and negative inputs of the opamp in the closed- 212 loop configurations) and the output voltage versus the $\mathbf{2 1 3}$ input voltage for the fault-free opamp operating in 214 three configurations were determined by simulation. 215 Our HSPICE simulations of these configurations are $\mathbf{2 1 6}$ shown in Figs. 3-5, respectively.

HSPICE simulations of the input offset voltage and 218 the output voltage for each fault group with respect to 219 the input voltage are shown in Figs. 6-9, respectively. 220 


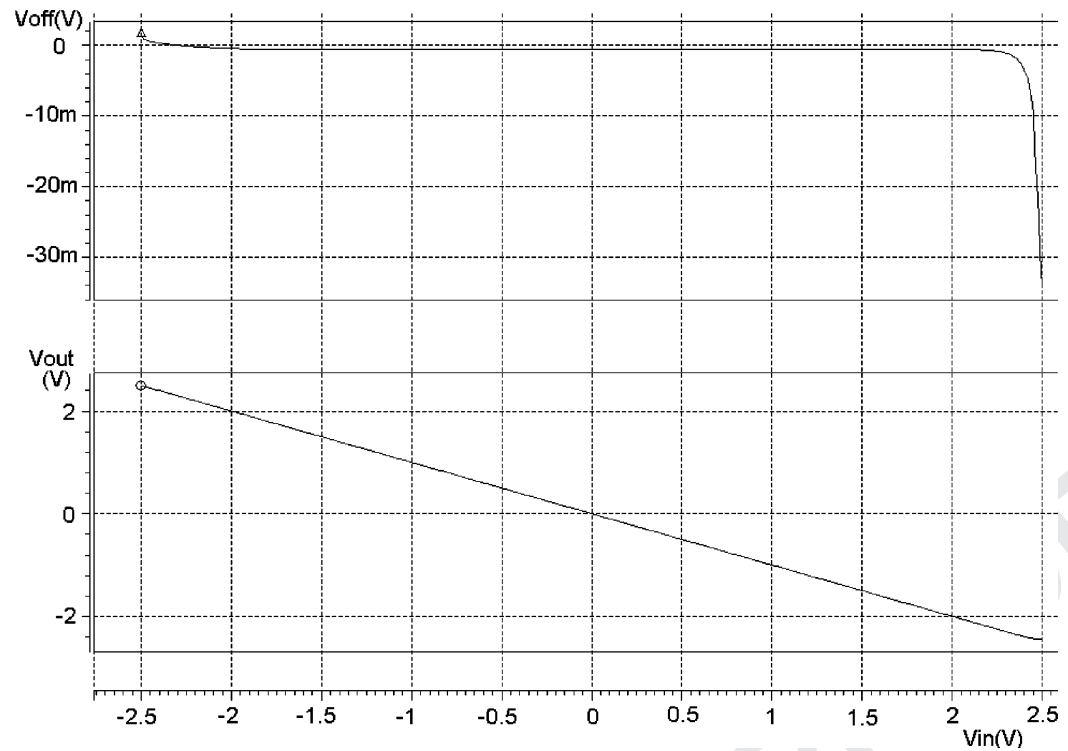

Fig. 3. Input offset voltage and output voltage versus DC-sweep input voltage for the fault-free inverting amplifier.

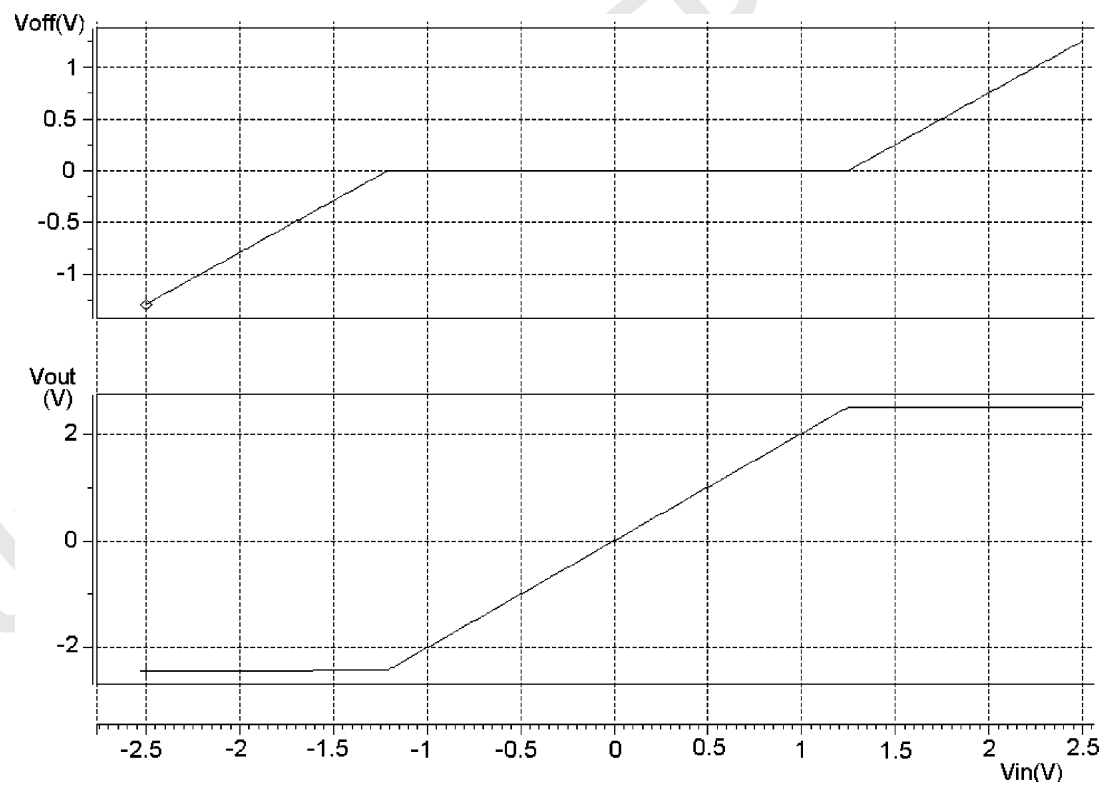

Fig. 4. Input offset voltage and output voltage versus DC-sweep input voltage for the fault-free non-inverting amplifier.

221 As can be seen from Figs. 7 and 9, the output re222 sponses of Type II and Type IV faults are quite sim223 ilar to the fault-free responses given in Figs. 3 and 4. 224 Type II and Type IV input offset voltages are notice225 ably different from the fault-free responses. The input 226 offset voltage has a small DC level for Type II faults, 227 but has a non-linear characteristic for Type IV faults.
The remaining two faults have very different char- $\mathbf{2 2 8}$ acteristics to the fault-free equivalents for both input $\mathbf{2 2 9}$ offset voltages and output voltages. It can be concluded $\mathbf{2 3 0}$ from the figures that a Type I fault causes the inverting $\mathbf{2 3 1}$ amplifier output to be "nearly stuck-at" a negative volt- $\mathbf{2 3 2}$ age near to the negative supply voltage level. A Type III $\mathbf{2 3 3}$ fault causes the inverting amplifier output to have a $\mathbf{2 3 4}$ 


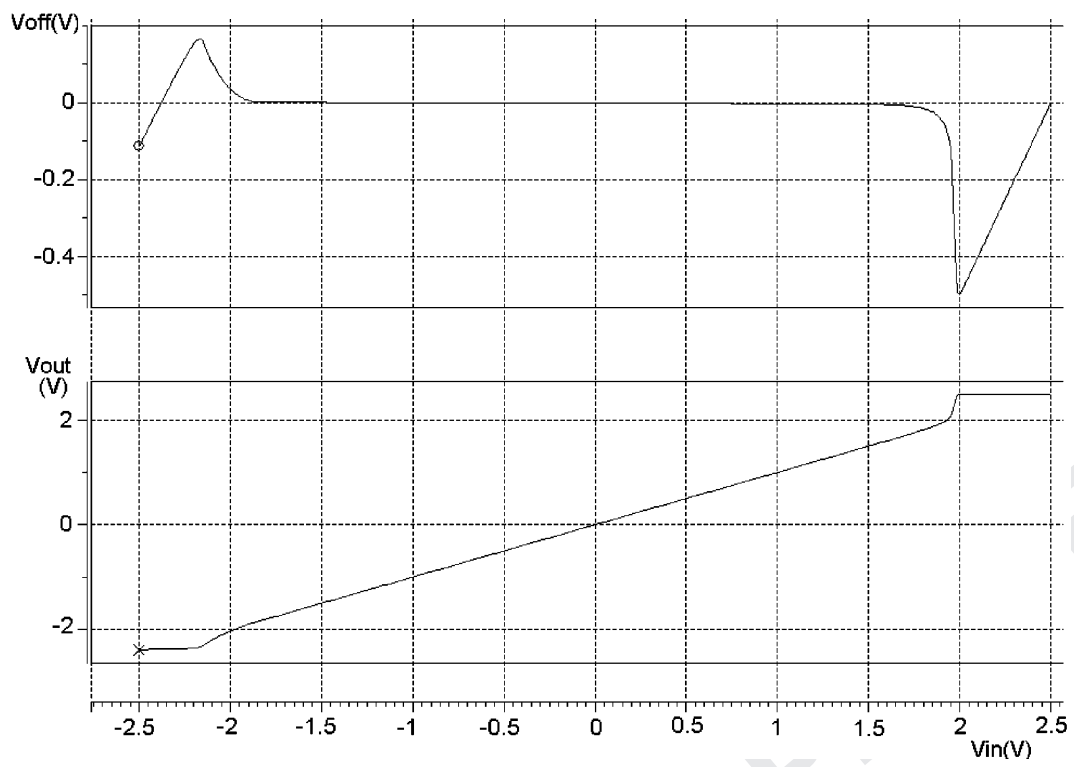

Fig. 5. Input offset voltage and output voltage versus DC-sweep input voltage for the fault-free unity gain buffer.

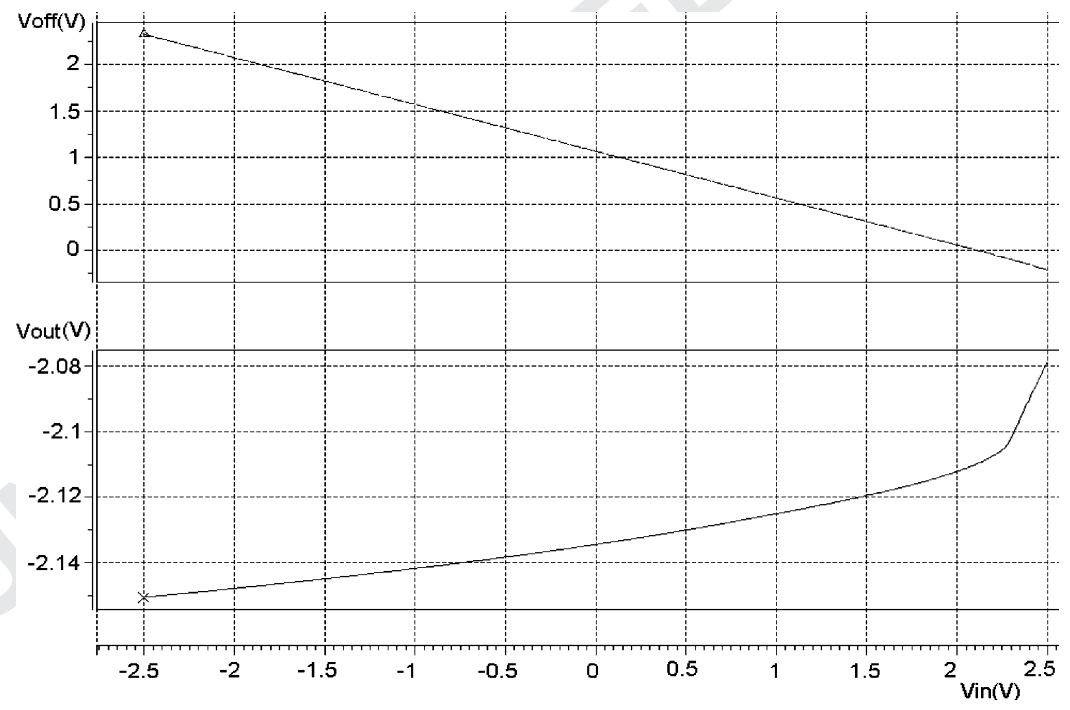

Fig. 6. Input offset voltage and the output voltage for the Type I fault (M4 drain-to-gate short fault for the inverting amplifier configuration).

235 non-inverting characteristic for the negative values of the DC input signal, and an inverting characteristic for

237 the positive values of the DC input signal. As can be

238 seen from the figures, the input offset voltage at the in-

239 puts of the opamp has a linear characteristic for Type I

240 faults, and a piecewise linear characteristic for Type III

241 faults.

242 The macromodel given in Fig. 10 for the inverting 243 opamp can be used to derive the input output relation- ship under fault conditions [10]:

$$
V_{\text {out }}=A_{\mathrm{CL}}\left[(1+m) V_{\text {in }}+k\right]
$$

where $A_{\mathrm{CL}}$ is the closed-loop gain for the opamp, the $\mathbf{2 4 5}$ parameters $m$ and $k$ are given in [10] as:

$$
m=\frac{-R 2}{D+R 2}
$$


P1: GIU

182 Kiliç and Zwoliński

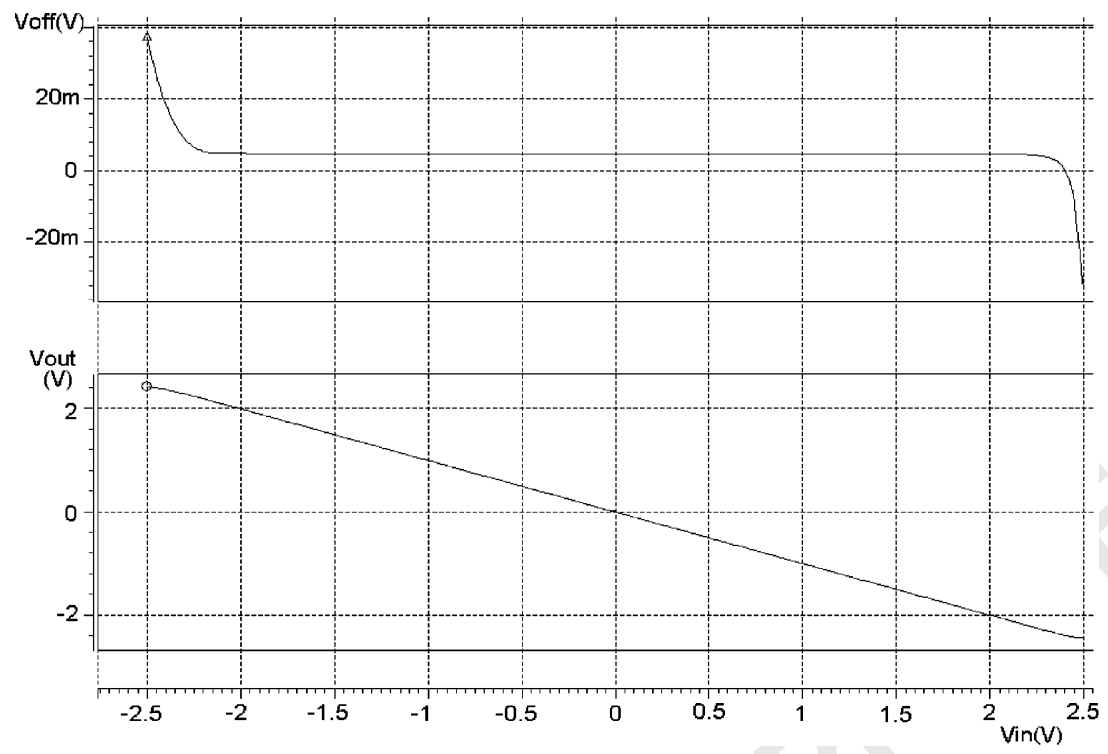

Fig. 7. Input offset voltage and the output voltage for the Type II fault (M5 drain-to-source short fault for the inverting amplifier configuration).

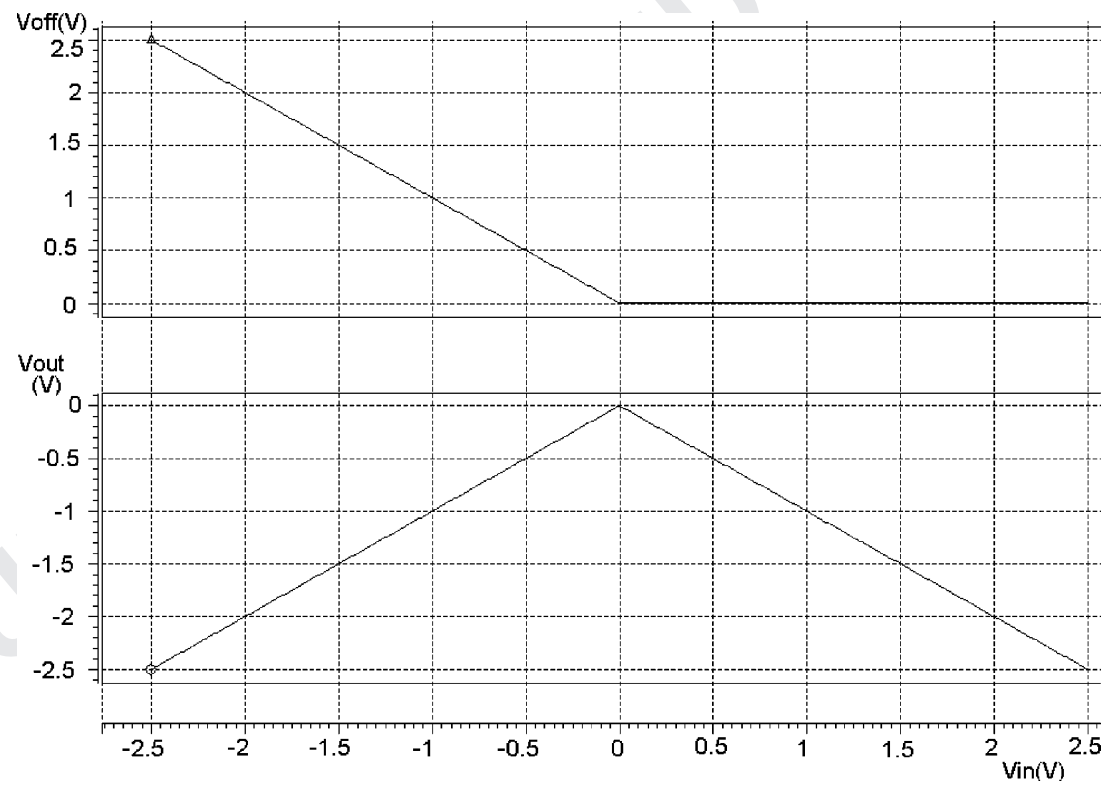

Fig. 8. Input offset voltage and the output voltage for the Type III fault (M6 open drain fault for the inverting amplifier configuration).

247 and

$$
k=a V o s+b V d d+c V s s
$$

248 where

$$
\begin{aligned}
& D=B(R 2 / / R o / / R d d / / R s s), \\
& B=\left(\frac{A}{R o}-\frac{1}{R 2}\right)(\text { Rid } / / R 1 / / R 2 / / 2 \text { Ricm }),
\end{aligned}
$$

$$
\begin{aligned}
a & =\frac{R 2 / / D}{A_{\mathrm{CL}}(R 1 / / R 2 / / 2 \text { Ricm } / / B R 2)}, \\
b & =\frac{S F}{A_{\mathrm{CL}} R d d}, \\
c & =-\frac{S F}{A_{\mathrm{CL}} R s s}, \\
A_{\mathrm{CL}} & =-\frac{R 2}{R 1},
\end{aligned}
$$




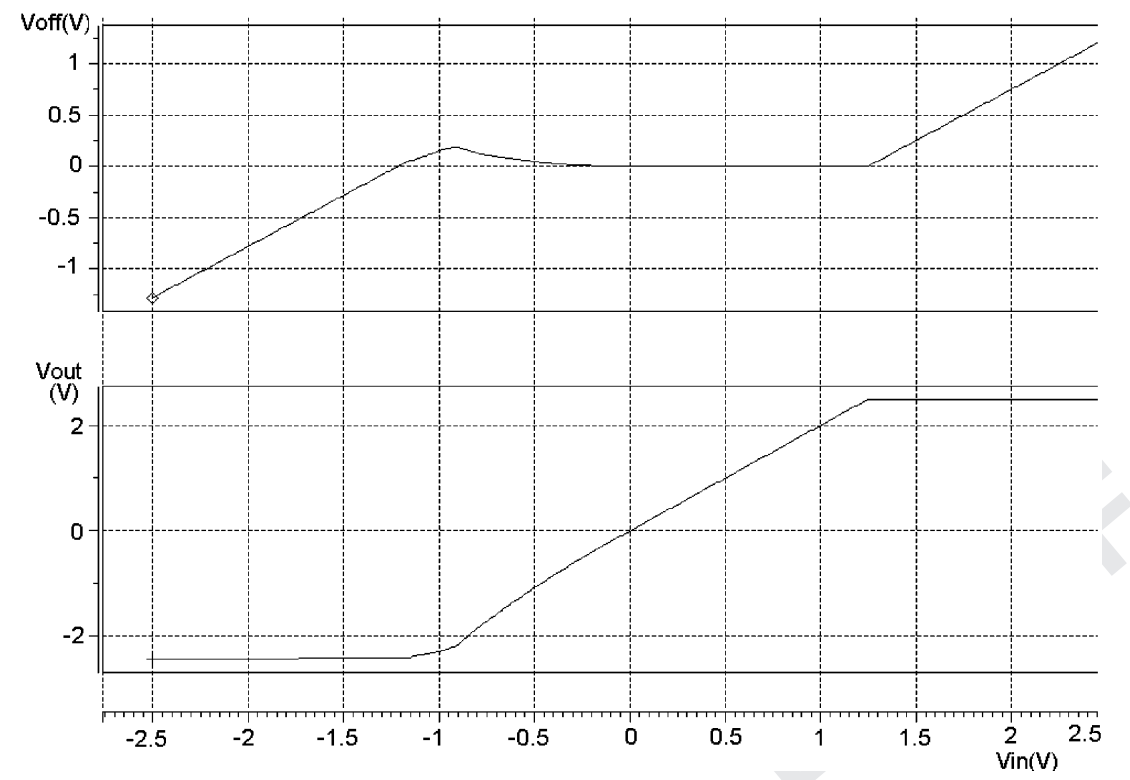

Fig. 9. Input offset voltage and the output voltage for the Type IV fault (M5 drain-to-source short fault for the non-inverting amplifier configuration).

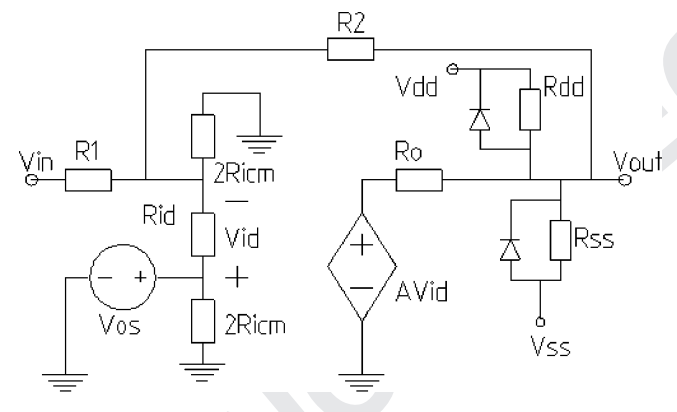

Fig. 10. Macromodel used in [10] to derive the input-output relationship for the closed loop inverting opamp.

$$
\begin{aligned}
S F= & R d d / / R s s / / / R o / /(R 2 / / R 11) / / \\
& \times \frac{\operatorname{Ro}(R 11 / / R 2)}{A R 11}, \\
R 11= & R 1 / / 2 \text { Ricm } / / \text { Rid },
\end{aligned}
$$

and $A$ represents the open-loop gain.

The non-ideal effects such as the input offset voltage, Vos, the finite open-loop gain, $A$, and the finite input and output resistances, Rid (differential mode input resistance), Ricm (common mode input resistance), Ro (output resistance), and the resistances from the output node to the supply rails ( $R d d$ and $R s s$ ) to model output stuck-at faults were taken into account when deriving
Eq. (1). Note that for the fault-free case Rid, Ricm, 257 $R d d, R s s$, and $A$ would be infinite, Vos, and Ro would 258 be zero, hence $m \rightarrow 0$, and $k \rightarrow 0$. When a fault causes 259 the output to be stuck-at some voltage level, $D \rightarrow 0, \mathbf{2 6 0}$ therefore $m \rightarrow-1$, and $k$ is the value of the stuck-at $\mathbf{2 6 1}$ output voltage; the closed-loop gain, $A_{\mathrm{CL}}$, is assumed $\mathbf{2 6 2}$ to be unity. As they are dealt with elsewhere [10], the $\mathbf{2 6 3}$ derivation of the above equations will not be given here. 264

The current limiting effect was also modeled in [10]. 265 This is due to the finite supply voltage at the output of $\mathbf{2 6 6}$ the opamp. It is claimed that the model covers all the $\mathbf{2 6 7}$ parametric faults and $92.5 \%$ of the catastrophic faults $\mathbf{2 6 8}$ that were considered. The model could not model the $\mathbf{2 6 9}$ M4 drain-to-gate short, M5 drain-to-source short, M1 270 open-gate faults for the non-inverting amplifier and the $\mathbf{2 7 1}$ M2 drain-to-gate short, M4 drain-to-gate short, M5 272 drain-to-source short, M1 open gate, M3 open source 273 and M5 open gate faults for the unity gain buffer.

\section{Behavioral Modeling Using HDLs}

HDLs have been in use for behavioral modeling and $\mathbf{2 7 6}$ simulation of digital circuits as well as analog elec- 277 tronic systems, fluid concentrations in chemical pro- $\mathbf{2 7 8}$ cesses, and even parachute jumps since 1960 [25]. 279 Currently two of the most widely used standards for $\mathbf{2 8 0}$ 
modeling digital designs are VHDL [26], and Verilog [27]. For analogue circuits, the choice has been between SPICE and proprietary analog HDLs.

Analog HDLs support the description of systems of differential and algebraic equations (DAEs). The solution of these systems varies continuously with time. Most analog HDLs support both structural composition and conservation semantics, in addition to behavioral descriptions. Examples of such languages are FAS [28], SpectreHDL [29], and Verilog-A [30].

Mixed-signal design has depended on the use of separate HDLs for the analog and digital parts or, again, on proprietary languages. Mixed-signal languages support both event-driven techniques and DAEs in one simulator. Simulators in this category are MAST/Saber [31], VeriasHDL [31], AdvanceMS [28], hAMSter [4].

Both VHDL and Verilog have been extended to analog mixed-signal design: VHDL-AMS [3], and Verilog-AMS [30]. The analog extensions to VHDL and Verilog should alleviate the multiple-language problem [32].

Since VHDL-AMS was standardized in 1999 there has been some work done on fault modeling using VHDL-AMS. One reason for the limited progress is, perhaps, that there is not yet a robust VHDL-AMS simulator available that has all the VHDL-AMS constructs implemented, such as procedural statements. Perkins et al. attempted to use an analog VHDL for fault modeling and simulation with limited success [1]. The HDLA modeling language with the ELDO simulator from Anacad (now a part of Mentor) was used. Behavioral model simulation using HDL-A and ELDO was over 4.6 times slower than the macromodel simulation carried out using HSPICE [1]. One of the reasons for this is that the semiconductor device models implemented in ELDO were not as efficient as those were in HSPICE.

\section{VHDL-AMS Behavioral Fault Model for the Inverting Opamp}

A VHDL-AMS model for the behavioral model given in Eq. (1) has been developed. The values of $m$ and $k$ were derived by carrying out transistor level simulations for four fault types and are given in Table 1.

Considering only the input-output relationship given in Eq. (1), the opamp macromodel given in Fig. 10 can now be simplified to that shown in Fig. 11. All the fault effects and non-ideal effects are approximated to Fos $=$
Table 1. The values of $m$ and $k$ for different fault groups.

\begin{tabular}{llc}
\hline & \multicolumn{2}{c}{ Parameters } \\
\cline { 2 - 3 } Fault types & \multicolumn{1}{c}{$m$} & $k(\mathrm{~V})$ \\
\hline Type I & -1.02 & 2.15 \\
Type II & 0 & 0.011 \\
Type III & 0 if vin $>0 \mathrm{~V}$ & 0 \\
& -2 if vin $<0$ & \\
Type IV & -1 if vin $>\sim 1.2 \mathrm{~V}$ & $\mathrm{~V}_{\mathrm{dd}} / 2$ if vin $>\sim 1 . \mathrm{V}$ \\
& $\quad$ and $\operatorname{vin}<\sim-1.2 \mathrm{~V}$ & $\mathrm{~V}_{\mathrm{ss}} / 2$ if $\operatorname{vin}<\sim-1.2 \mathrm{~V}$ \\
& 0 if $\sim-1.2 \mathrm{~V}$ & 0 if $\sim-1.2 \mathrm{~V}$ \\
& $<$ vin $<\sim 1.2 \mathrm{~V}$ & $<$ vin $<\sim 1.2 \mathrm{~V}$ \\
\hline
\end{tabular}

$m V_{\text {in }}+k$, which is applied to the inverting input of the $\mathbf{3 2 8}$ opamp.

A VHDL-AMS implementation of the behavioral $\mathbf{3 3 0}$ model given in Eq. (1) is shown in Figs. 12 and 13331 [33]. rin represents the input resistance of the opamp 332 in Fig. 12 where it is only used for the third equation $\mathbf{3 3 3}$ in Fig. 13. The third equation is needed as there are 334 three quantities declared in the architecture declaration 335

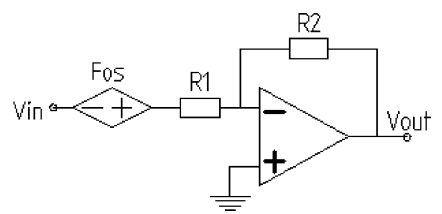

Fig. 11. Behavioral level DC-offset fault model for the inverting opamp.

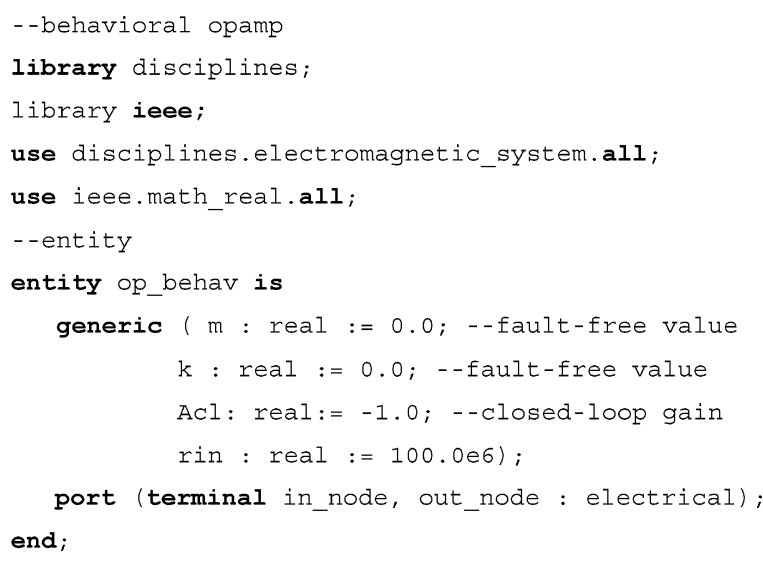

Fig. 12. The VHDL-AMS entity implementation of the behavioral fault model. 
336

337

338

339

340

341

342 shown in Fig. 13. Note that this architecture declaration also covers the supply voltage limiting effect at the output of the opamp.

In order to simulate the VHDL-AMS model shown in Figs. 12 and 13, one also needs VHDL-AMS models for a resistor, a voltage source and a testbench, which are shown in Figs. 14-16, respectively.

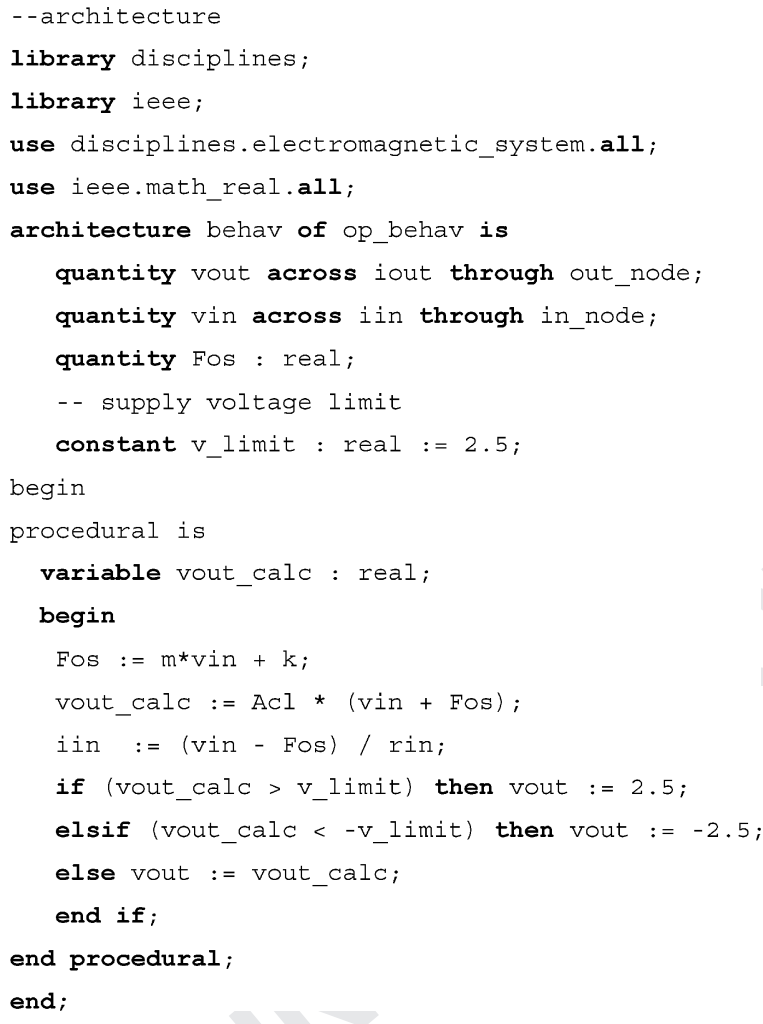

Fig. 13. The VHDL-AMS architecture implementation of the behavioral fault model.

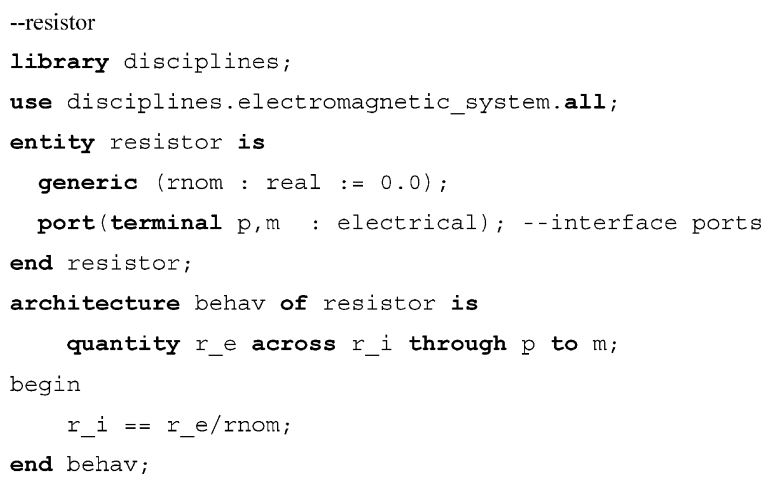

Fig. 14. A VHDL-AMS model of a resistor.

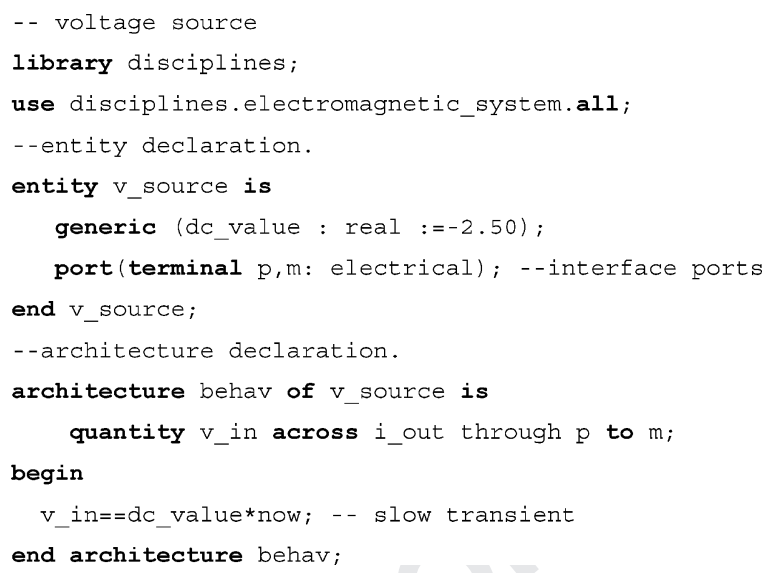

Fig. 15. A VHDL-AMS model of a voltage source.

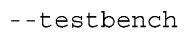

Fig. 16. A VHDL-AMS testbench used with the hAMSter simulator to simulate the behavioral model shown in Figs. 12 and 13.

Note that input voltage source in the architecture $\mathbf{3 4 3}$ declaration shown in Fig. 15 is realized using a pre- $\mathbf{3 4 4}$ defined VHDL-AMS function, now, which returns the $\mathbf{3 4 5}$ value of the current time at each step as simulation pro- $\mathbf{3 4 6}$ ceeds. This is done in order to simulate the DC-sweep $\mathbf{3 4 7}$ analysis, which is not defined in VHDL-AMS (unlike $\mathbf{3 4 8}$ many SPICE-like simulators). This technique is called $\mathbf{3 4 9}$ slow transient simulation. 
351

\section{Simulation Results}

352 hAMSter). Figs. 19-26.
The slow transient simulation results using the hAMSter simulator and the behavioral closed-loop VHDLAMS model of the inverting opamp (the fault free case) with the necessary component and voltage source models and the testbench given in the previous sections are shown in Figs. 17 and 18.

Note that the X-axis in Figs. 17 and 18 represents the time in seconds, where Y-axis represents vout, vin, and Fos in Volts. (Unless otherwise stated, for the rest of the paper it will be assumed that $\mathrm{X}$-axis will represent time in seconds for the simulation results obtained using

Using the values for the parameters $m$ and $k$ from Table 1 yields the simulation results shown in
Note that the output response of the opamp, vout, $\mathbf{3 6 7}$ found for the positive values of vin and the negative $\mathbf{3 6 8}$ values of vin for Type I faults (Figs. 19 and 20) are the $\mathbf{3 6 9}$ same ("nearly stuck-at" -2.14 V), as expected. $\quad 370$

For Type III faults Fos is determined using the fol- 371 lowing if-then construct in the VHDL-AMS model. 372

For Type IV faults Fos is determined using the fol- 373 lowing if-then construct in the VHDL-AMS model. 374

DC-sweep analysis cannot be performed for VHDL- 375 AMS. Therefore, the transient simulation results for $\mathbf{3 7 6}$ different fault types using the VHDL-AMS behav- 377 ioral models and the hAMSter simulator were com- $\mathbf{3 7 8}$ pared with the transient simulation results obtained us- 379 ing transistor level models with HSPICE simulator. To $\mathbf{3 8 0}$ do that a sine wave with $2 \mathrm{~V}$ peak-to-peak magnitude 381 and $1 \mathrm{KHz}$ frequency was applied to both behavioral 382 and transistor level circuits. The simulators were run

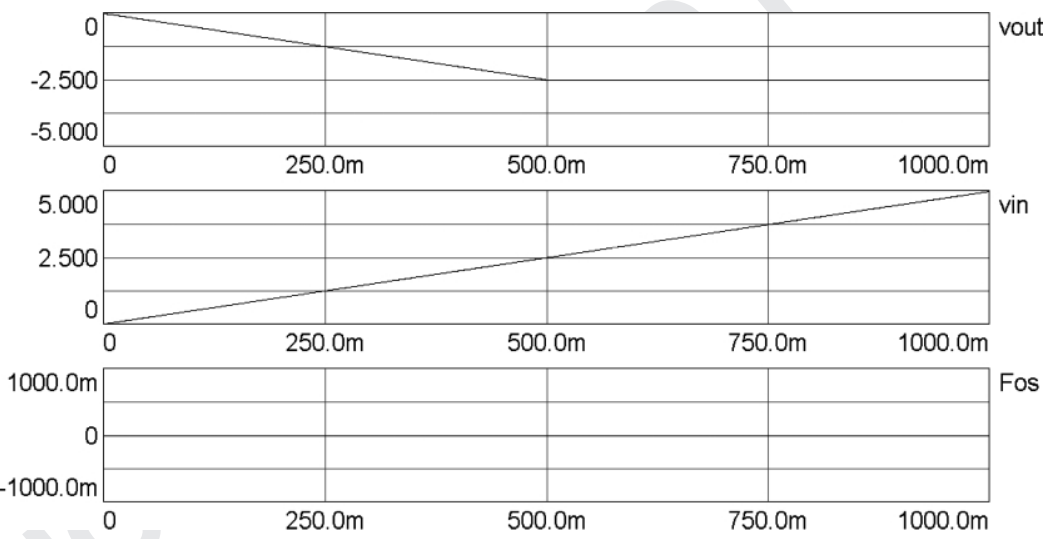

Fig. 17. Slow-transient simulation results using the VHDL-AMS model with hAMSter for the positive values of the input voltage source.

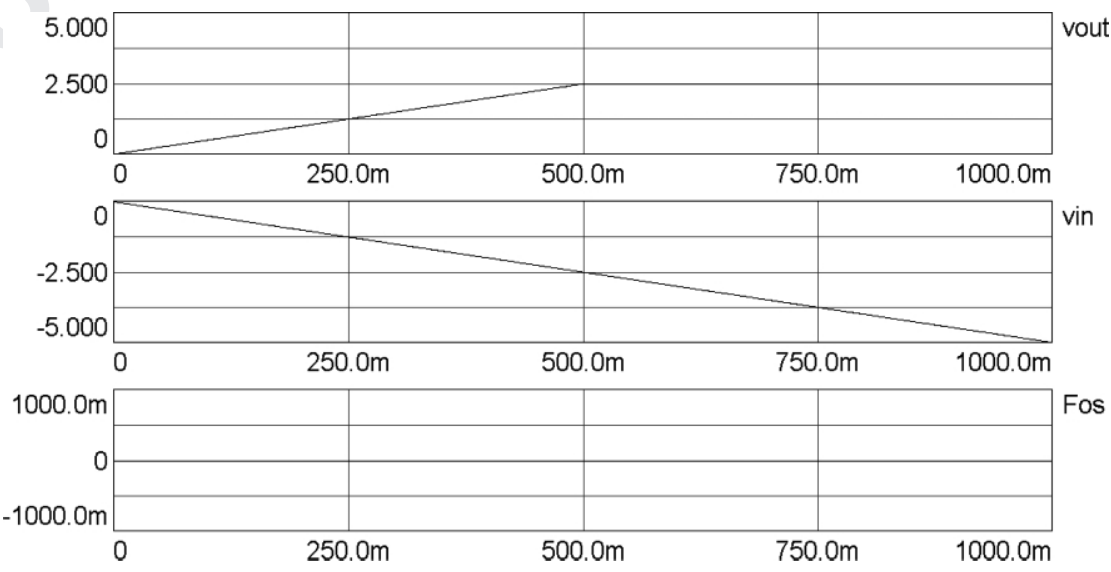

Fig. 18. Slow-transient simulation results using the VHDL-AMS model with hAMSter for the negative values of the input voltage source. 
P1: GIU

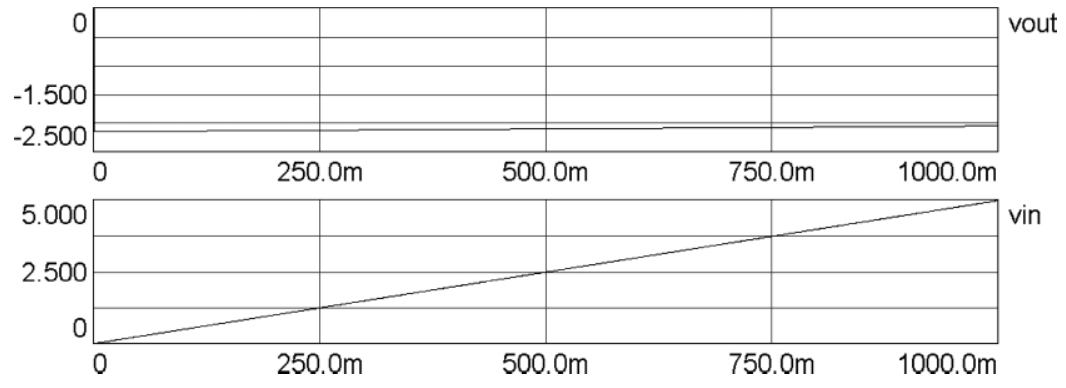

Fig. 19. Slow-transient simulation using hAMSter for Type I faults for the positive values of vin.

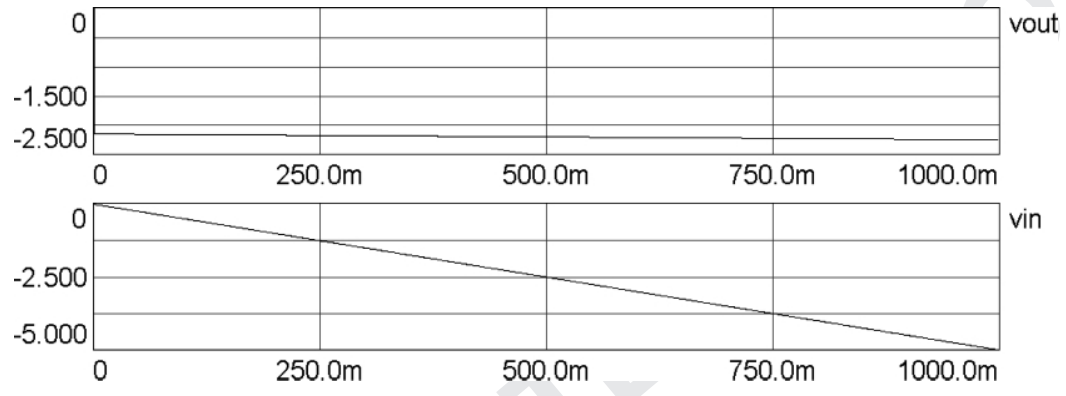

Fig. 20. Slow-transient simulation using hAMSter for Type I faults for the negative values of vin.

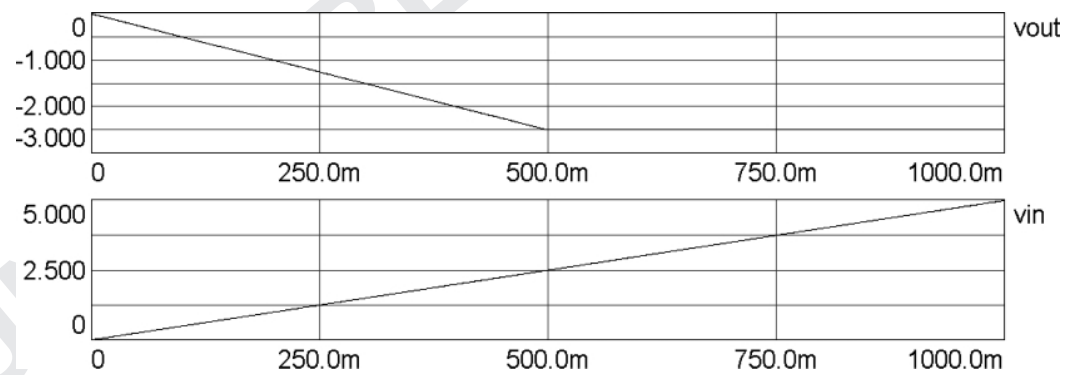

Fig. 21. Slow-transient simulation using hAMSter for Type II faults for the positive values of vin.

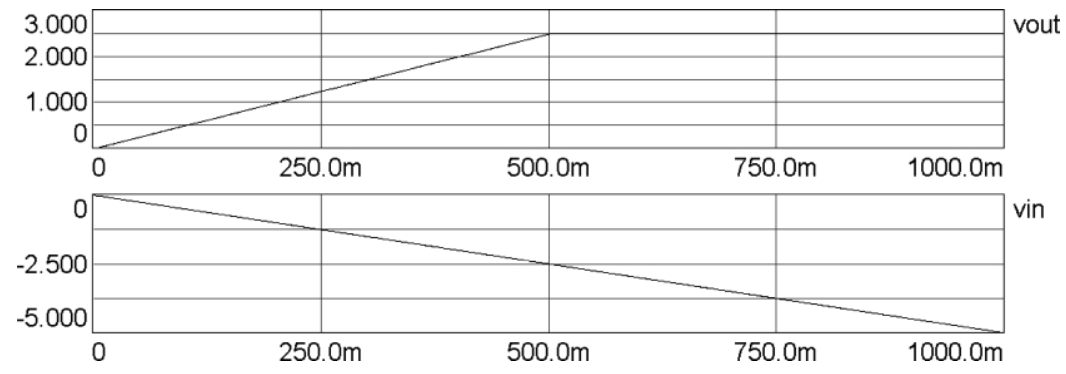

Fig. 22. Slow-transient simulation using hAMSter for Type II faults for the negative values of vin. 
P1: GIU

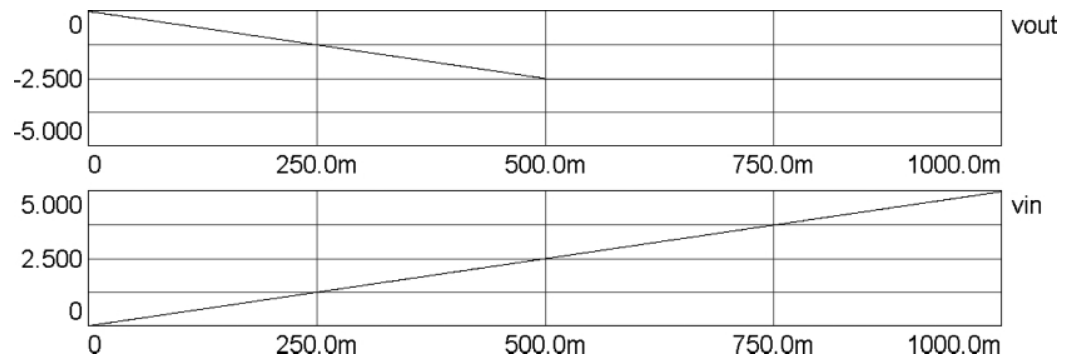

Fig. 23. Slow-transient simulation using hAMSter for Type III faults for the positive values of vin.

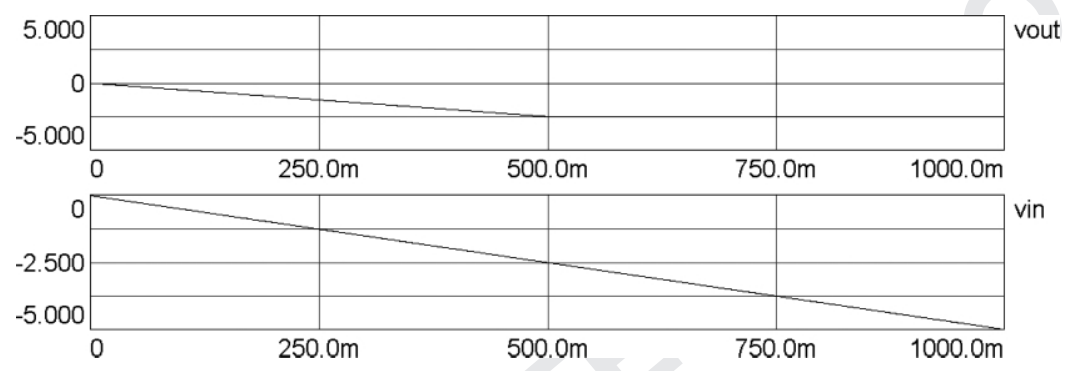

Fig. 24. Slow-transient simulation using hAMSter for Type III faults for the negative values of vin.

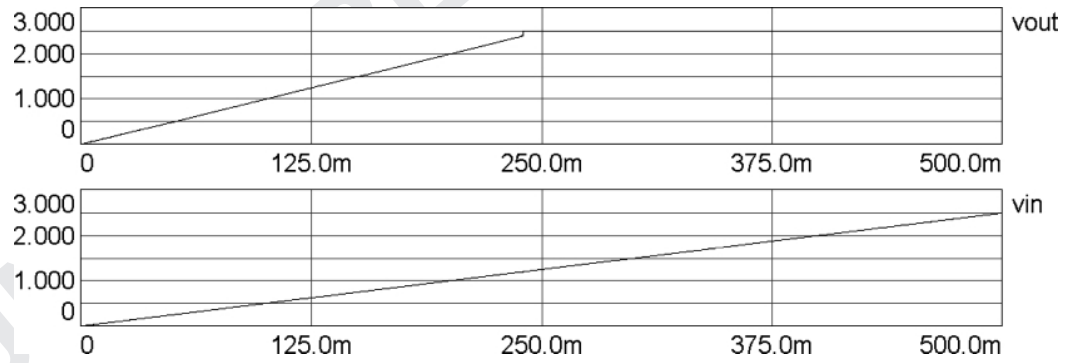

Fig. 25. Slow-transient simulation using hAMSter for Type IV faults for the positive values of vin.

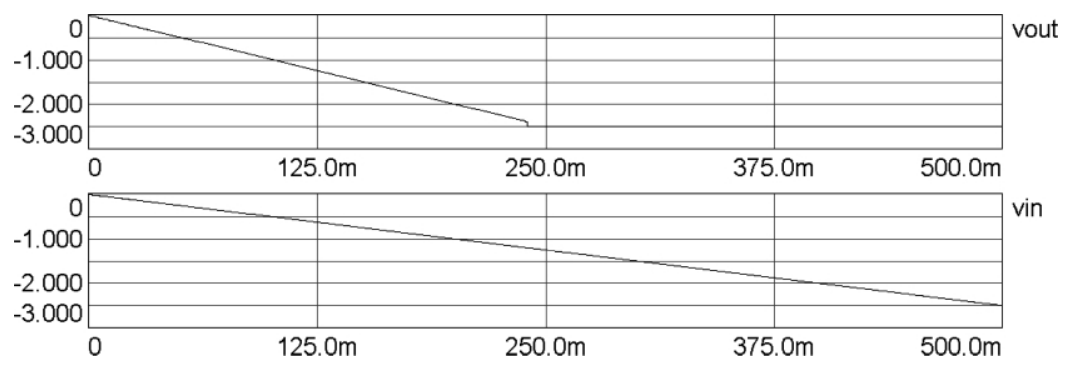

Fig. 26. Slow-transient simulation using hAMSter for Type IV faults for the negative values of vin. 
Table 2. Comparison of CPU times for transistor level transient HSPICE simulations against VHDL-AMS behavioral level hAMSter simulations.

\begin{tabular}{|c|c|c|}
\hline \multirow[b]{2}{*}{ Fault type } & \multicolumn{2}{|c|}{$\begin{array}{l}\text { The CPU time (s) } \\
\text { Simulator }\end{array}$} \\
\hline & hAMSter & HSPICE \\
\hline Fault I & $90 \mathrm{~m}$ & $400 \mathrm{~m}$ \\
\hline Fault II & $90 \mathrm{~m}$ & $360 \mathrm{~m}$ \\
\hline Fault III & $100 \mathrm{~m}$ & 37.37 \\
\hline Fault IV & $140 \mathrm{~m}$ & $350 \mathrm{~m}$ \\
\hline
\end{tabular}

$\mathrm{Au}:$ Pls. cite Fig. 27 in the text.

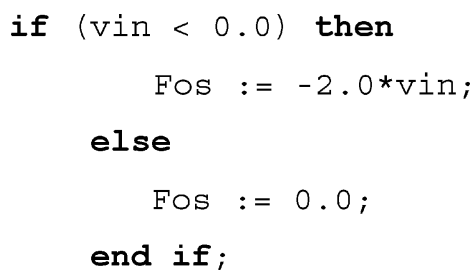

Fig. 27. if-then construct implemented in the VHDL-AMS model for Type III faults.

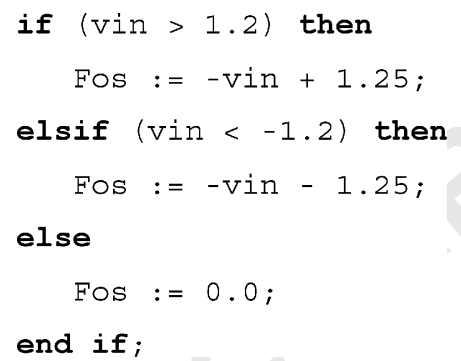

Fig. 28. If-then construct implemented in the VHDL-AMS model for Type IV faults.

383 tion of the if-then construct required in the procedural 397 statement (Fig. 28) to model the Type IV faults.

\section{Conclusions}

Capturing circuit behavior under faulty conditions at a $\mathbf{4 0 0}$ higher level using mathematical equations (behavioral 401 modeling) is somewhat simpler than the macromodel $\mathbf{4 0 2}$ approach.

403

Analog fault simulation is a key factor in $\mathbf{4 0 4}$ analog/mixed-signal test generation. Currently such $\mathbf{4 0 5}$ fault simulation is of limited use due to the speed of $\mathbf{4 0 6}$ analog simulation and the large number of faults to $\mathbf{4 0 7}$ be simulated. Simulation can be speeded up by using $\mathbf{4 0 8}$ number of techniques. Behavioral modelling is one of 409 those techniques. We have shown in this paper how one $\mathbf{4 1 0}$ can increase analog fault simulation speed by using be- $\mathbf{4 1 1}$ havioral models. We have used VHDL-AMS for the $\mathbf{4 1 2}$ behavioral modelling. It is clear that as VHDL-AMS 413 simulators become more powerful it will be easier to $\mathbf{4 1 4}$ model analog/mixed-signal circuits at a higher level so $\mathbf{4 1 5}$ as to speed-up simulation in general and analog fault $\mathbf{4 1 6}$ simulation in particular.

\section{References}

1. A.J. Perkins, M. Zwolinski, C.D. Chalk, and B.R. Wilkins, 419 "Fault modeling and simulation using VHDL-AMS." Analog In- $420 \mathrm{Au}$ : Pls. tegrated Circuits and Signal Processing, vol. 16, pp. 141-155, 421 verify pp. 53-67, 1998.

2. M. Abramovici, M.A. Breuer, and A.D. Friedman, Digital Sys- 423range. tems Testing and Testable Design, IEEE Press, 1990.

3. www.eda.org/analog $\mathbf{4 2 5}$

4. http://www.hamster-ams.com/ $\mathbf{4 2 6}$

5. C.-Y. Pan and K.-T. Cheng, "Fault macro modeling for 427 analog/mixed-signal circuits," in IEEE International Test Con- $\mathbf{4 2 8}$ ference, ITC'97, 1997, pp. 913-922.

6. G. Casinovi and A. Sangiovanni-Vincentelli, "A macromodeling 430 algorithm for analog circuits." IEEE Transactions on Computer- 431 Aided Design of Integrated Circuits and Systems, vol. 10, no. 2, 432 pp. 150-160, 1991.

7. B. Perez et al., "A new nonlinear time-domain op-amp macro- 434 model using threshold functions and digitally controlled network $\mathbf{4 3 5}$ elements." IEEE Journal of Solid-State Circuits, vol. 23, no. 4, 436 pp. 959-971, 1988.

437

8. G.A. Boyle, D.O. Pederson, B.M. Cohn, and J.E. Solomon, 438 "Macromodeling of integrated circuit operational amplifiers." 439 IEEE J. of Solid State Circuits, vol. SC-9, pp. 353-363, 1974.440

9. M.E. Brinson and D.J. Faulkner, "Modular SPICE macromodel 441 for operational amplifiers." IEE Proc.- Circuits Devices Syst., $\mathbf{4 4 2}$ vol. 141, no. 5, pp. 417-420, 1994.

10. Y.-J. Chang et al., "A behavior-level fault model for the closed- 444 loop operational amplifier." Journal of Information Science and $\mathbf{4 4 5}$ Engineering, vol. 16, no. 5, pp. 751-766, 2000. 
11. C. Chalk and M. Zwolinski, "Macromodel of CMOS operational amplifier including supply current variation." Electronics Letters, vol. 31, pp. 1398-1400, 1995.

12. P. Mandal and V. Visvanathan, "Macromodeling of the AC characteristics of CMOS op-amps," in IEEE 1993 Conference on Computer Aided Design, Digest of Technical Papers, 1993, pp. 334-339.

13. M.E. Brinson and D.J. Faulkner, "A SPICE noise macromodel for operational amplifiers." IEEE Transactions on Circuits and Systems-I: Fundamental Theory and Applications, vol. 42, no. 3, pp. 166-168, 1995.

14. G. Krajewska and F.E. Holmes, "Macromodeling of FET/bipolar operational amplifiers." IEEE Journal of Solid-State Circuits, vol. SC-14, no. 6, pp. 1083-1087, 1979.

15. C. Turchetti and G. Masetti, "A macromodel for integrated allMOS operational amplifiers." IEEE Journal of Solid-State Circuits, vol. SC-18, pp. 389-394, 1983.

16. M.E. Brinson and D.J. Faulkner, "SPICE macromodel for operational amplifier power supply current sensing." Electronics Letters, vol. 30, no. 23, pp. 166-168, 1994.

17. R.V. Peic, "Simple and accurate nonlinear macromodel for operational amplifiers." IEEE Journal of Solid-State Circuits, vol. 26, no. 6, pp. 896-899, 1991.

18. B. Al-Hashimi, "Behavioural simulation of filters." IEE Colloquium on Analogue Simulation: The Dream \& The Nightmare, pp. 51-55, 1995.

19. A.I. Kayssi and K.A. Sakallah, "Macromodel simplification using dimensional analysis," in 1994 Int. Symp. on Circuits and Systems, 1994, pp. 335-338.

20. M. Zwolinski, Z.R. Yang, and T.J. Kazmierski, "Using robust adaptive mixing for statistical fault macromodelling." IEE Proceedings: Circuits, Devices and Systems, vol. 147, no. 5, pp. 265-270, 2000

21. G. Casinovi, "Multi-level simulation of large analog systems containing behavioral models." IEEE Transactions on Computer Aided Design of Integrated Circuits and Systems, vol. 13, no. 11, pp. 1391-1399, 1994.

22. E. Bruls et al., "Analogue fault simulation in standard VHDL." IEE Proc. Circuits Devices Syst., vol. 143, no. 6, pp. 380-385, 1996.

23. C.G. Broyden et al., "A class of methods for solving nonlinear simultaneous equations," Mathematics of Computation, vol. 19, no. 92 , pp. 577-593, 1965.

24. http://www.ee.washington.edu/mad/benchmarks/benchmarks. html

25. E. Christen and K. Bakalar, "VHDL-AMS, A hardware description language for analog applications." IEEE Transactions on Circuits and Systems-II: Analog and Digital Signal Processing, vol. 46. no. 10, pp. 1263-1272, 1999.

26. VHDL Language Reference Manual, IEEE Standard 10761993.

27. Standard Description Language Based on the Verilog ${ }^{\mathrm{TM}}$ Hardware Description Language, IEEE Standard 13641995.

28. www.mentor.com

29. www.cadence.com

30. www.verilog.com

31. www.analogy.com

32. www.ednmag.com
33. Y. Kilic, "Testing techniques and fault simulation for analogue $\mathbf{5 0 6}$ CMOS integrated circuits." University of Southampton, PhD $\mathbf{5 0 7}$ Thesis, Chapter 6, 2001.

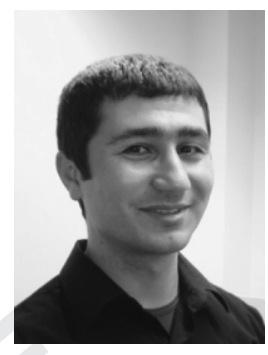

Yavuz Kılıç gained his B.Sc. (Hons) (electronics and 509 telecommunications), M.Sc. (microelectronics) from $\mathbf{5 1 0}$ Y1ldız Technical University of Istanbul (Turkey), and $\mathbf{5 1 1}$ Ph.D. from the University of Southampton (UK) in $\mathbf{5 1 2}$ 1994, 1996, and 2001, respectively. He is currently with $\mathbf{5 1 3}$ Allegro MicroSystems Europe Ltd., Scotland (UK) as $\mathbf{5 1 4}$ a senior design engineer. Prior to this, he has held po- $\mathbf{5 1 5}$ sitions with Cyan Technology, Cambridgeshire (UK) $\mathbf{5 1 6}$ and Philips Semiconductors, Southampton (UK) as a $\mathbf{5 1 7}$ design engineer, and at the University of Southamp- $\mathbf{5 1 8}$ ton (UK) and Yıldız Technical University (Turkey) 519 as a Research Assistant. His current interests include $\mathbf{5 2 0}$ mixed-signal CMOS/BiCMOS integrated circuit de- $\mathbf{5 2 1}$ sign, simulation, test, and power management related $\mathbf{5 2 2}$ IC design. He is a member of the IEEE Circuits and $\mathbf{5 2 3}$ Systems Society.



Mark Zwolinski received the B.Sc. and Ph.D. 525 degrees in electronics from the University of $\mathbf{5 2 6}$ Southampton, UK, in 1982 and 1986, respectively. He 527 is a Senior Lecturer in the Department of Electron- 528 ics and Computer Science, University of Southampton, $\mathbf{5 2 9}$ UK. His research interests include behavioral synthe- $\mathbf{5 3 0}$ sis, design for test, fault simulation and VHDL. He is $\mathbf{5 3 1}$ chair of the IEEE DATC subcommittee on fault simu- $\mathbf{5 3 2}$ lation. He has co-authored about 100 research papers $\mathbf{5 3 3}$ in technical journals and conferences. 